\title{
Violência e vulnerabilidade no território do agente comunitário de saúde: implicações no enfrentamento da COVID-19
}

\author{
Violence and vulnerability of the Community Health Worker \\ in the territory: implications for tackling COVID-19
}

Anya Pimentel Gomes Fernandes Vieira-Meyer (http://orcid.org/0000-0003-4237-8995) ${ }^{1}$

Ana Patrícia Pereira Morais (http://orcid.org/0000-0001-6188-7897) ${ }^{2}$

Isabella Lima Barbosa Campelo (http://orcid.org/0000-0003-1552-7350) ${ }^{3}$

José Maria Ximenes Guimarães (http://orcid.org/0000-0002-5682-6106) ${ }^{2}$

${ }^{1}$ Fundação Oswaldo Cruz - Ceará. R. São José, s/n. 61760-000 Eusébio CE Brasil.

anyavieira10@gmail.com

${ }^{2}$ Universidade Estadual do Ceará. Fortaleza CE Brasil.

${ }^{3}$ Centro Universitário Fanor. Fortaleza CE Brasil.

\begin{abstract}
The scope of the study was to investigate the relationship between COVID-19 and social vulnerability variables in Fortaleza, as well as to verify the influence of violence on the work of the Community Health Worker (ACS) and the implications for tackling COVID-19. Primary quantitative data (e.g., questionnaires with the $A C S$ ) and secondary data (e.g., HDI, homicide, illiteracy, Bolsa Família [PBF] coverage and Family Health Strategy [ESF] coverage) were used. There was a relationship between COVID-19 and indicators of social vulnerability, where cases had a negative relationship with HDI and illiteracy; and deaths had a positive relationship with the homicide rate and PBF coverage. Regions with high death rates due to COVID-19 and social vulnerability have greater FHS coverage and less home visits by the CHA. The latter's role in tackling COVID-19 is limited by the violence in the territory. The thorough performance of the ACS in confronting this pandemic is dependent on intersectoral policies. Thus, the creation of a policy to tackle COVID-19 with the participation of the ACS needs to consider intersectoral actions for curtailing and preventing violence in the territory, in addition to adequate training in the prevention and detection of COVID-19.
\end{abstract}

Key words Violence, Primary Health Care, Vulnerable population groups, Community health workers, COVID-19
Resumo Objetivou-se averiguar a relação da COVID-19 com variáveis de vulnerabilidade social em Fortaleza e verificar a influência da violência no trabalho do ACS e suas implicações no enfrentamento da COVID-19. Dados quantitativos primários (e.g., questionários com ACS) $e$ secundários (e.g., IDH, homicídio, analfabetismo, cobertura do Programa Bolsa Família [PBF] e da Estratégia Saúde da Família [ESF]) foram utilizados. Observou-se relação da COVID-19 com indicadores de vulnerabilidade social, onde os casos têm relação negativa com IDH e analfabetismo; e os óbitos relação positiva com taxa de homicídios e cobertura do PBF. Regiões com altas taxas de óbito por COVID-19 e vulnerabilidade social possuem maior cobertura da ESF e menor realização de visita domiciliar pelo ACS. A atuação deste, no enfrentamento da pandemia, é limitada pela violência do território. A plena atuação do ACS, no enfrentamento da COVID-19, é dependente de políticas intersetoriais. Assim, a construção de uma política de enfrentamento da COVID-19, com participação do ACS, precisa levar em consideração, além de seu adequado treinamento na prevenção e detecção de COVID-19, ações intersetoriais para o enfrentamento e prevenção da violência no território.

Palavras-chave Violência, Atenção Primária à Saúde, Populações Vulneráveis, Agentes Comunitários de Saúde, COVID-19 


\section{Introdução}

A doença emergente COVID-19, declarada pandemia em março de 2020 ao atingir 24 países ${ }^{1}$, alcança todos os continentes e representa um grave problema de saúde pública global. No Brasil, o primeiro caso foi anunciado em 26 de fevereiro de 2020, expandindo-se para 1.864 .681 casos e 72.100 óbitos até 12 de julho de 2020, sendo o terceiro país mais afetado no globo ${ }^{2}$. Evidencia-se que apenas cinco estados brasileiros respondem por $81 \%$ das ocorrências de óbitos: São Paulo, Rio de Janeiro, Ceará, Pernambuco e Amazonas, que refletem a heterogeneidade geográfica e social do país ${ }^{3}$.

A pandemia de COVID-19 tem sido um desafio para países que apresentam profundas desigualdades sociais. Com efeito, descortinou as imensas desigualdades econômicas, sociais, políticas e culturais existentes no mundo e, particularmente, no Brasil. A doença afeta mais intensamente os grupos populacionais mais vulneráveis, entre estes os idosos, os negros, os pobres e pessoas em situação de rua. Assim, destacam-se as possíveis influências de variáveis, como território, violência, racismo, condições de habitação, condições de trabalho, renda e desigualdade de acesso a bens e serviços, incluindo-se os serviços de saúde, no perfil de morbimortalidade por COVID-194-6.

Para além do cenário epidemiológico, convém assinalar as possíveis repercussões da $\mathrm{CO}-$ VID-19 nos índices de violência e criminalidade, historicamente maiores em cenários de vulnerabilidades sociais, os quais podem ser agravados pelo desemprego e pelo isolamento social. Nesse contexto, observou-se o aumento da violência doméstica contra mulheres, crianças e adolescentes $^{7}$, bem como ocorreu aumento das taxas de homicídios ${ }^{8,9}$. Contudo, ainda não se tem estudos que analisem as possíveis relações entre a violência e o enfrentamento da COVID-19 nos territórios de vulnerabilidade.

No enfrentamento à pandemia, países com sistemas universais de saúde têm melhor capacidade de resposta por oferecer cobertura universal de serviços aos cidadãos, desde a promoção da saúde e a prevenção até o tratamento de alta complexidade $^{10}$. No Brasil, o Sistema Único de Saúde (SUS) é uma importante política pública de saúde, estruturada com base na Atenção Primária à Saúde (APS), operacionalizada no nível municipal pela Estratégia Saúde da Família (ESF), que, desde 1994, oferta cuidados interdisciplinares à população brasileira. A ESF é orga- nizada por uma equipe composta por médico, enfermeiro, cirurgião-dentista, técnico/auxiliar de enfermagem, técnico ou auxiliar de saúde bucal e Agentes Comunitários de Saúde (ACS) ${ }^{11}$. As diretrizes clínicas para a atuação da APS nos casos de COVID-19, estabelecem ações de teleatendimento, medidas de prevenção, identificação de suspeitos e estratificação de gravidade da doença, bem como manejo terapêutico e isolamento domiciliar dos casos leves ${ }^{12}$. Nesse cenário, os ACS que atuam nos territórios, salvo aqueles inseridos nos grupos de risco e respeitando-se as medidas de prevenção, devem realizar as visitas domiciliares como importante ferramenta para informar, fazer busca ativa de suspeitos e acompanhamento de $\operatorname{casos}^{13}$.

Os ACS favorecem o acesso aos serviços de saúde, mediando a construção de vínculos entre as famílias e as equipes da ESF/APS, considerando seu trabalho no território, fortemente realizado por meio de visitas domiciliares, nas quais se realizam coleta de dados sobre as condições de vida e saúde das famílias, mas também fornecem orientações e apoio na resolução de demandas junto aos membros da equipe de saúde. Com efeito, destacam-se as evidências de que a atuação dos ACS melhora os resultados de saúde em várias condições e contextos ${ }^{14}$, o que denota seu potencial de intervenção no enfrentamento da COVID-19. Todavia, esses profissionais estão sujeitos a várias questões de contexto presentes antes e durante a pandemia, que podem influenciar seu processo de trabalho na comunidade, a exemplo da violência e da situação de pobreza das famílias.

O município de Fortaleza, que concentra o maior volume de casos da COVID-19 no Ceará, tem registrado um crescente número de casos desde a introdução do vírus até a manutenção dos casos autóctones, chegando a 37.835 casos confirmados e 3.581 óbitos até 10 de julho de $2020^{15}$. No âmbito da sua rede de APS, conta com 113 unidades básicas de saúde, nas quais atuam 416 equipes da ESF e 2.093 ACS $^{16}$. Esses, conforme a Política Nacional de Atenção Básica ${ }^{11}$, atuam, principalmente, em visitas domiciliares, ações de promoção da saúde e de cunho intersetorial, como o Programa Saúde na Escola (PSE), e foram, recentemente, inseridos nas ações de enfrentamento à COVID-19 $9^{12,13}$.

Não obstante, o município registra marcante desigualdade social expressa nas condições de moradia, trabalho e renda, além de altos índices de violência. Fortaleza situa-se entre as capitais mais violentas do Nordeste ${ }^{17}$, e foi considerada a 
nona cidade mais violenta do mundo, em $2018^{18}$. Porém, são limitados os estudos nesta capital averiguando a relação entre variáveis de vulnerabilidade e a morbimortalidade por COVID-19, assim como a relação da violência com o trabalho dos ACS, importantes atores no enfrentamento dessa pandemia. Com base nessa problemática, este estudo tem por objetivos averiguar a relação de casos e óbitos por COVID-19 com variáveis de vulnerabilidade social no município de Fortaleza-CE e verificar a influência da violência no trabalho do ACS e suas implicações no enfrentamento da COVID-19.

\section{Metodologia}

\section{Dados}

A presente pesquisa baseia-se em dados primários e secundários. Os dados primários foram coletados, no período entre junho e novembro de 2019, por meio de questionários aplicados aos Agentes Comunitários de Saúde (ACS) no município de Fortaleza. Este questionário é parte do projeto de pesquisa "Saberes e práticas de Agentes Comunitários de Saúde sobre a violência urbana no território e as repercussões em seu trabalho e sua saúde", aprovado pelo Comitê de Ética em Pesquisa da Universidade de Fortaleza. Entre as perguntas realizadas, incluiu-se o tempo que trabalha como ACS; as atividades desenvolvidas na função de ACS (Realiza visita domiciliares, Realiza atividades de Promoção da Saúde em grupos específicos [e.g., grávidas, alunos, diabéticos, hipertensos, idosos etc.], Realiza atividades do Programa Saúde na Escola); o ACS se sente capacitado para lidar com a violência na comunidade em que trabalha; e informações sociodemográficas (e.g., idade e gênero).

Os dados secundários (taxa de homicídio, Índice de Desenvolvimento Humano [IDH], Cobertura do Programa Bolsa Família [PBF], casos e óbitos por COVID-19, taxas de analfabetismo, taxa de cobertura da ESF) foram obtidos junto aos órgãos e/ou documentos oficiais do município de Fortaleza.

$\mathrm{O}$ número total de casos e óbitos pela $\mathrm{CO}$ VID-19, nos bairros de Fortaleza, foram extraídos dos informes semanais da COVID-19 da Célula de Vigilância Epidemiológica, vinculada à Coordenadoria de Vigilância em Saúde, publicados no site da Prefeitura Municipal de Fortaleza, Ceará (https://coronavirus.fortaleza.ce.gov.br/ boletim-epidemiologico.html). O IDH foi extraí- do do documento da Secretaria Municipal de Desenvolvimento Econômico de Fortaleza, denominado Desenvolvimento Humano, por bairro, em Fortaleza, publicado em 2014 (salasituacional. fortaleza.ce.gov.br:8081). O número de homicídios anual dos últimos 5 e 10 anos, o número de habitantes e os dados de analfabetismo para a faixa etária 10-14 anos, por bairro de Fortaleza, foram obtidos junto à Célula de Vigilância Epidemiológica, da Secretaria de Saúde de Fortaleza. Os dados de cobertura do Programa Bolsa Família (PBF) foram obtidos na coordenação técnica do Programa Bolsa Família de Fortaleza, ligada à Secretaria dos Direitos Humanos e Desenvolvimento Social (SDHDS) do município. Dados da cobertura da ESF para as regionais de saúde de Fortaleza foram fornecidos pela Célula de Atenção Primária à Saúde do município.

Com base nos dados, calculou-se, para cada bairro de Fortaleza, a taxa de cobertura do PBF, a taxa de analfabetismo para a faixa etária 10-14 anos, a taxa de homicídio nos últimos 5 anos (2015-2019) e nos últimos 10 anos (2010-2019), a taxa de casos de COVID-19 em três tempos (T1-04.05.2020; T2-29.05.2020 e T3-19.06.2020) e a taxa de óbitos de COVID-19 nos referidos tempos. Importante ressaltar que o tempo 1 (T1) apresenta os dados desde o início da pandemia até a data 04.05.2020, nos tempos 2 e 3 (T2 e T3) foram considerados os casos acumulados desde o início da pandemia até a data dos informes semanais. Ademais, para o T3, também se dividiu os bairros em três grupos para as taxas de casos e óbitos por COVID-19 (Baixo, Médio e Alto). Cada um desses grupos com cerca de um terço do número de bairros de Fortaleza.

\section{Análise dos dados}

Correlação de Spearman, com seus respectivos intervalos de confiança, foi utilizada para verificar a relação entre as taxas de casos e óbitos da COVID-19 nos três tempos analisados e as seguintes variáveis (relacionadas à vulnerabilidade populacional): taxa de homicídio dos últimos 5 anos, taxa de homicídio dos últimos 10 anos; IDH, taxa de analfabetismo 10-14 anos; cobertura do Programa Bolsa Família. Como múltiplas correlações foram realizadas, utilizou-se a correção de Bonferonni para o nível de significância. De acordo com a Prefeitura Municipal de Fortaleza, o município possui 119 bairros (https:// www.fortaleza.ce.gov.br/a-cidade), distribuídos em seis Secretarias Regionais (SER), que exercem a gestão descentralizada de políticas setoriais, 
como saúde e educação, entre outras. Contudo, alguns dados não estavam disponíveis para todos os bairros e em outros disponíveis apenas para as SER (e.g., cobertura da ESF).

Para analisar a relação entre taxa de casos e óbitos por COVID-19 e a realização ou não das atividades desenvolvidas pelos ACS, calculou-se a média das respostas dadas pelos ACS em cada um dos bairros. Como as respostas originais eram binárias ( $0=$ Não e $1=S i m)$, obteve-se uma variável contínua variando de zero a um, onde quanto maior o valor, maior era a proporção de ACS, que realizavam aquela atividade no referido bairro.

Posteriormente, tendo como unidade de análise o ACS, avaliou-se a relação entre as atividades realizadas na função de ACS e as taxas de violência nos últimos 5 e 10 anos nos bairros de Fortaleza utilizando o teste t. A frequência da resposta dada pelos ACS em relação à variável "se sente capacitado para lidar com a violência na comunidade em que trabalha" foi calculada, sendo testada a possibilidade de influência do gênero, da idade e do tempo como ACS, com a utilização dos testes t e qui-quadrado.

Calculou-se, para cada uma das regionais de saúde, a proporção de crescimento das taxas de óbito e casos de COVID-19. Para a proporção de crescimento da taxa de óbito por COVID-19, o cálculo foi feito subtraindo a taxa média de óbitos em T1 da taxa média em T3, dividindo este valor pela taxa média em T1 e multiplicando por 100. Cálculo similar foi feito para proporção de crescimento da taxa de casos, mas utilizando as taxas de casos de COVID-19 por regional. Além disso, comparou-se a cobertura da ESF, IDH, taxa de cobertura do PBF, a proporção de crescimento das taxas de óbito e casos de COVID-19, e as taxas de homicídio nos últimos 5 e 10 anos nas diferentes regiões de saúde, utilizando o teste one way anova (ANOVA). Nível de significância foi definido em $\mathrm{p} \leq 0,05$.

\section{Resultados}

Um total de 1.498 ACS responderam ao questionário. Desses, 1.239 trabalhavam no mesmo bairro em que moravam, sendo estes os que foram analisados no presente estudo. As taxas de óbitos e casos por COVID-19 variaram entre 0 e 596,13, e entre 38,13 e $5.067,06$, respectivamente. As taxas de homicídio variaram entre 3,79 e 297,26. O IDH teve média de 0,305 , variando entre 0,119 e 0,953. A taxa de analfabetismo (10-14 anos) teve média de 2,99 , variando entre 0,37 e 7,9 . A taxa de cobertura da ESF variou entre 41,86 na regional II de Fortaleza e 80,67, na regional V. A taxa de cobertura do PBF variou entre 22,54 a 30,79. Todas as taxas foram calculadas por $100 \mathrm{mil} \mathrm{ha-}$ bitantes.

As correlações entre as variáveis de casos e óbitos de COVID-19 e as variáveis de vulnerabilidade (Tabela 1) demonstram uma relação dos casos de COVID-19 com IDH e taxa de analfabetismo de 10-14 anos. A relação com IDH é positiva com os três tempos analisados, enquanto a correlação com taxa de analfabetismo é negativa nos T2 (29.05.2020) e T3 (19.06.2020). Assim, os casos aumentam em locais com IDH mais altos e com taxas de analfabetismo mais baixas, mostrando uma relação inversa com variáveis relacionadas à vulnerabilidade social.

Por outro lado, a taxa de óbitos por COVID-19 se correlaciona positivamente com a cobertura do PBF (nos três tempos estudados), ficando mais forte (aumento de $r$ ) com o passar do tempo. Correlação positiva também foi observada entre taxa de óbitos por COVID-19 e as taxas de homicídios nos últimos 5 (T3) e 10 anos (T2 e T3). Em relação à taxa de homicídios nos últimos 10 anos, observa-se, também, um aumento da correlação (aumento de $r$ ) com o passar do tempo (Tabela 1). Assim, os óbitos por COVID-19 demonstram uma relação positiva com variáveis relacionadas à vulnerabilidade social.

Investigou-se, assim, tendo como base as regionais de saúde, a cobertura da ESF (onde os ACS se inserem profissionalmente), IDH, taxa de analfabetismo 10-14 anos, taxa de cobertura do PBF e as taxas de homicídio nos últimos 5 e 10 anos nas diferentes regionais de saúde de Fortaleza (Tabela 2). Identificou-se diferenças entre as regionais (SER) em todas as variáveis analisadas (one-way anova). Notou-se, também que, de uma maneira geral, as SER V e VI possuem as maiores taxas de homicídio nos últimos 5 e 10 anos, menores IDH, maiores taxas de cobertura do PBF, maiores taxas de analfabetismo (valores mais altos nas SER V, I e VI), maiores taxas de cobertura da ESF (valores mais altos nas SER V, III e VI) e maiores proporções de crescimento das taxas de óbitos e de casos de COVID-19 nos tempos estudados (valores mais altos nas SER IV, V e VI) (Tabela 2). Desse modo, os locais com maior percentual de ACS (cobertura ESF) são, de maneira geral, aqueles com variáveis relacionadas à maior vulnerabilidade social e maior crescimento de casos e óbitos por COVID-19 (Figura 1, Tabela 2), colocando o ACS como um potencial ator no combate à pandemia, principalmente com ações 
Tabela 1 Correlação entre taxas de óbitos e casos de COVID-19 com variáveis relacionadas a vulnerabilidade socioeconômica. Fortaleza, Ceará, Brasil.

\begin{tabular}{|c|c|c|c|c|c|c|c|}
\hline & & $\begin{array}{c}\text { Taxa Óbitos } \\
\text { COVID T1 } \\
(\mathbf{0 4 . 0 5 . 2 0 2 0 )}\end{array}$ & $\begin{array}{c}\text { Taxa Casos } \\
\text { COVID T1 } \\
(\mathbf{0 4 . 0 5 . 2 0 2 0 )}\end{array}$ & $\begin{array}{c}\text { Taxa Óbitos } \\
\text { COVID T2 } \\
(29.05 .2020)\end{array}$ & $\begin{array}{c}\text { Taxa Casos } \\
\text { COVID T2 } \\
(29.05 .2020)\end{array}$ & $\begin{array}{c}\text { Taxa Óbitos } \\
\text { COVID T3 } \\
(19.06 .2020) \\
\end{array}$ & $\begin{array}{c}\text { Taxa Casos } \\
\text { COVID T3 } \\
(19.06 .2020)\end{array}$ \\
\hline \multirow{3}{*}{$\begin{array}{l}\text { Taxa Homicídio } \\
\text { últimos } 5 \text { anos }\end{array}$} & 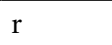 & 0,2643 & 0,0730 & 0,2926 & 0,1167 & $0,3590\left(^{*}\right)$ & 0,1813 \\
\hline & $\mathrm{p}^{*}$ & 0,3111 & $>0,9999$ & 0,1067 & $>0,9999$ & 0,0055 & $>0,9999$ \\
\hline & & $0,087-0,425$ & $-0,110-0,251$ & $0,117-0,450$ & $-0,066-0,292$ & $0,190-0,507$ & $-0,000-0,351$ \\
\hline \multirow{3}{*}{$\begin{array}{l}\text { Taxa Homicídio } \\
\text { últimos } 10 \text { anos }\end{array}$} & $\mathrm{r}$ & 0,2763 & 0,0485 & $0,3160\left(^{*}\right)$ & 0,1459 & $0,3768\left(^{*}\right)$ & 0,1893 \\
\hline & $\mathrm{p}^{*}$ & 0,1999 & $>0,9999$ & 0,0405 & $>0,9999$ & 0,0022 & $>0,9999$ \\
\hline & 95\%IC & $0,100-0,436$ & $(-) 0,134-0,228$ & $0,143-0,471$ & $(-) 0,037-0,319$ & $0,210-0,523$ & $0,008-0,358$ \\
\hline \multirow[t]{3}{*}{ IDH } & $\mathrm{r}$ & 0,0978 & $0,4366\left(^{\star}\right)$ & 0,0641 & $0,5196\left(^{*}\right)$ & $-0,0390$ & $0,4885\left(^{*}\right)$ \\
\hline & $\mathrm{p}$ & $>0,9999$ & 0,0001 & $>0,9999$ & $<0,0001$ & $>0,9999$ & $<0,0001$ \\
\hline & 95\%IC & $-0,084-0,274$ & $0,278-0,572$ & $-0,118-0,242$ & $0,374-0,640$ & $-0,218-0,143$ & $0,338-0,615$ \\
\hline Taxa & $\mathrm{r}$ & $-0,0861$ & $-0,3065$ & $-0,0951$ & $-0,3976$ & $-0,0690$ & $-0,4131$ \\
\hline Analf: & $\mathrm{p}^{*}$ & $>0,9999$ & 0,0575 & $>0,9999$ & 0,0006 & $>0,9999$ & 0,0003 \\
\hline $10-1$ & $95 \% \mathrm{IC}$ & $-0,263-0,096$ & $-0,462--0,133$ & $-0,271-0,087$ & $-0,540--0,234$ & $-0,247-0,113$ & $-0,553--0,251$ \\
\hline & $\mathrm{r}$ & $0,3321\left(^{*}\right)$ & 0,2014 & $0,3215\left(^{\star}\right)$ & 0,1646 & $0,4805\left(^{*}\right)$ & 0,2537 \\
\hline Progr & $\mathrm{p}^{*}$ & 0,0175 & $>0,9999$ & 0,0283 & $>0,9999$ & $<0,0001$ & 0,4194 \\
\hline Famíliz & $95 \% \mathrm{IC}$ & $0,162-0,483$ & $0,022-0,368$ & $0,150-0,474$ & $-0,016-0,335$ & $0,329-0,608$ & $0,077-0,415$ \\
\hline
\end{tabular}

${ }^{\star}$ Correlação de Spearman.

Tabela 2. Valores médios por regional de saúde (SER) das taxas de cobertura da ESF, homicídio nos últimos 5 e 10 anos, índice de desenvolvimento humano (IDH), taxa de analfabetismo 10-14anos, taxa de cobertura do PBF, taxas de óbito e casos de COVID-19 nos tempos T1 (04.05.2020), T2 (29.05.2020) e T3 (19.06.2020), assim como proporção de crescimento de óbitos e casos de COVID-19 entre T1 e T3. Fortaleza, Ceará, Brasil.

\begin{tabular}{lrrrrrrr}
\hline & SER I & SER II & SER III & SER IV & SER V & SER VI & p $^{*}$ \\
\hline Taxa de Cobertura Estratégia Saúde da Família & 67,44 & 41,86 & 79,44 & 43,36 & 80,67 & 77,02 & \\
Taxa Homicídio últimos 5 anos & 61,71 & 53,74 & 59,37 & 41,22 & 76,12 & 69,64 & $<0,001$ \\
Taxa Homicídio últimos 10 anos & 70,27 & 64,39 & 64,7 & 47,59 & 78,66 & 76,02 & $<0,001$ \\
Índice de Desenvolvimento Humano (IDH) & 0,299 & 0,450 & 0,298 & 0,411 & 0,237 & 0,275 & $<0,001$ \\
Taxa Analfabetismo 10-14 anos & 3,14 & 2,60 & 3,05 & 1,91 & 3,32 & 3,08 & $<0,001$ \\
Taxa de Cobertura Programa Bolsa Família & 22,54 & 22,94 & 27,75 & 20,19 & 37,30 & $30,79<0,001$ \\
Taxa Óbitos COVID-19 T1 (04.05.2020) & 37,31 & 41,94 & 20,86 & 18,03 & 16,30 & $16,39<0,001$ \\
Taxa Casos COVID-19 T1 (04.05.2020) & 254,3 & 385,09 & 192,44 & 216,74 & 157,57 & 234,17 & $<0,001$ \\
Taxa Óbitos COVID-19 T2 (29.05.2020) & 122,95 & 123,88 & 89,45 & 90,03 & 80,09 & $60,32<0,001$ \\
Taxa Casos COVID-19 T2 (29.05.2020) & 687,62 & 838,79 & 580,02 & 714,41 & 581,93 & $722,39<0,001$ \\
Taxa Óbitos COVID-19 T3 (19.06.2020) & 183,22 & 166,09 & 132,36 & 135,14 & 114,25 & $104,52<0,001$ \\
Taxa Casos COVID-19 T3 (19.06.2020) & 1099,60 & 1260,49 & 922,29 & 1153,85 & 972,84 & $1167,79<0,001$ \\
Proporção de crescimento óbitos COVID-19 & 391,07 & 296,02 & 534,52 & 649,53 & 600,92 & 537,71 & $<$ \\
(T1 a T3) & & & & & & & \\
Proporção de crescimento casos COVID-19 & 332,40 & 227,32 & 379,26 & 432,37 & 517,40 & 398,69
\end{tabular}

^ANOVA (comparação entre as regionais de saúde - SER).

de prevenção e monitoramento dos casos via visita domiciliar.

Selecionando-se como base de análise o bairro (no caso do ACS, seu bairro de moradia e trabalho), analisou-se a relação entre atividades desenvolvidas na função de ACS e taxa de casos e de óbitos por COVID-19 (aqui categorizada em baixa, média ou alta). Observou-se relação entre 


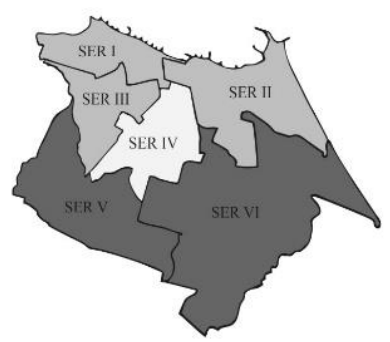

Legenda

Taxa Homicídios últimos 5 anos

SER_Fortaleza

41.6 a 53.1

53.1 a 64.6

64.6 a 76.1

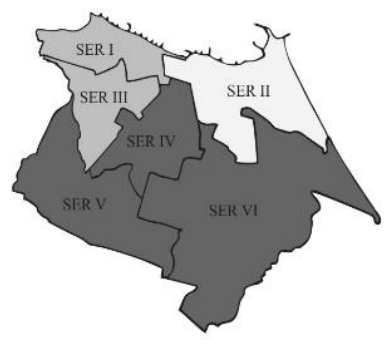

Legenda

Proporção Crescimento Caso COVID19

SER_Fortaleza

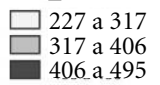

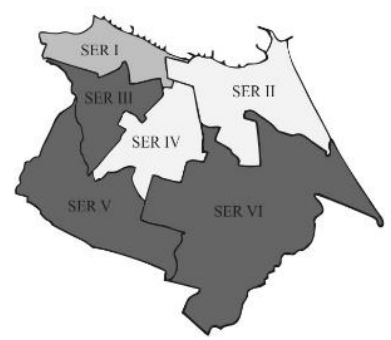

Legenda

Taxa Cobertura ESF

SER_Fortaleza

$\square 41.9$ a 54.8

54.8 a 67.7

64.6 a 76.1

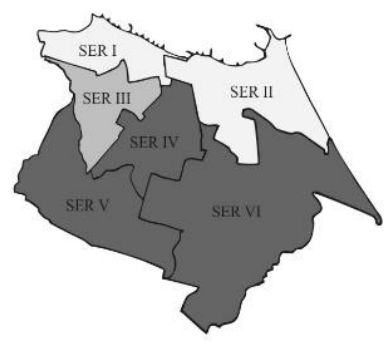

Legenda

Proporção Crescimento Óbitos COVID19

SER_Fortaleza

$\square 296$ a 417

417 a 538
538 a 495

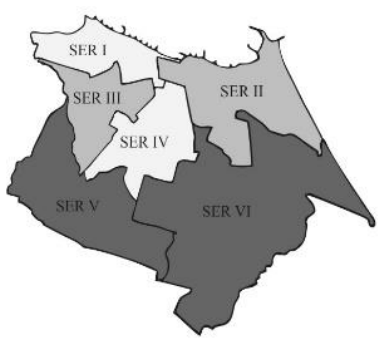

Legenda

Taxa Cobertura Programa Bolsa Família SER_Fortaleza

$\square 20.0$ a 22.8

22.8 a 28.3

28.3 a 37.3

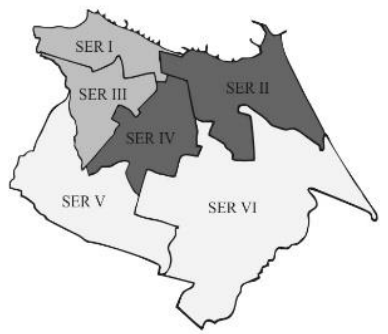

Legenda

Índice Desenvolvimento Humano SER_Fortaleza

$\square 0.239$ a 0.290

0.290 a 0.337

0.337 a 0.450

Figura 1. Mapas dos indicadores de vulnerabilidade social (Cobertura Programa Bolsa Família, Taxa de Homicídio dos últimos 5 anos, IDH) e as taxas de crescimento dos casos e óbitos por COVID19 das regiões de saúde do município de Fortaleza, Ceará, Brasil.

Fonte: Instituto Brasileiro de Geografia e Estatística; Secretaria Municipal de Saúde de Fortaleza; Secretaria de Desenvolvimento Econômico de Fortaleza; Secretaria dos Direitos Humanos e Desenvolvimento Social de Fortaleza.

taxa de óbitos por COVID-19 e as atividades desempenhadas pelos ACS. Nos bairros com alta taxa de óbitos por COVID-19, a proporção dos ACS que realizam visita domiciliar são mais baixas. Inversamente, a proporção de ACS que realizam atividades do Programa Saúde na Escola (PSE) é mais alta nos bairros com taxas de óbitos por COVID-19 mais elevadas. A realização de atividades de promoção da saúde não se relacionou com a taxa de óbitos por COVID-19 (Tabela 3). As taxas de casos de COVID-19 não se relacionaram com a realização de atividades dos ACS. Dessa forma, constatou-se que os ACS realizam me- nos visitas domiciliares e mais ações do PSE em locais com maior mortalidade por COVID-19.

Avaliando-se a relação entre taxas de homicídios nos últimos 5 e 10 anos com a realização de atividades do ACS, observou-se uma relação entre as taxas de homicídios e realizar visita domiciliar, em que as taxas de homicídios eram mais altas onde os ACS informaram não realizar visita domiciliar. Inversamente, observou-se relação entre realizar atividades do PSE e taxas de homicídios nos últimos 5 e 10 anos, onde taxas de homicídios foram mais altas em locais onde atividades desse programa eram realizadas (Tabela 4). 
Tabela 3. Valores médios de atividades desenvolvidas pelos Agentes Comunitários de Saúde (ACS) em seu trabalho junto a Estratégia Saúde da Família (ESF) por categoria de óbitos e casos de COVID-19 (baixo, médio ou alto) no tempo T3 (19.06.2020). Fortaleza, Ceará, Brasil.

\begin{tabular}{|c|c|c|c|c|c|c|c|}
\hline \multirow{2}{*}{\multicolumn{2}{|c|}{$\begin{array}{l}\text { Atividades dos Agentes } \\
\text { Comunitários de Saúde }\end{array}$}} & \multicolumn{3}{|c|}{$\begin{array}{c}\text { Taxa Óbitos COVID-19 T3 } \\
(19.06 .2020)\end{array}$} & \multicolumn{3}{|c|}{$\begin{array}{c}\text { Taxa Casos COVID-19 T3 } \\
(19.06 .2020)\end{array}$} \\
\hline & & Baixo & Médio & Alta & Baixo & Médio & Alta \\
\hline \multirow[t]{3}{*}{ Realiza Visita Domiciliares } & Média & 0,9767 & 0,9914 & 0,936 & 0,9614 & 0,975 & 0,9614 \\
\hline & $\mathrm{DP}$ & 0,051 & 0,023 & 0,085 & 0,068 & 0,0475 & 0,078 \\
\hline & $\mathrm{p}^{*}$ & 0,0005 & & & 0,5863 & & \\
\hline \multirow{3}{*}{$\begin{array}{l}\text { Atividades de Promoção } \\
\text { da Saúde }\end{array}$} & Média & 0,6887 & 0,6713 & 0,6756 & 0,7404 & 0,6766 & 0,6686 \\
\hline & DP & 0,314 & 0,252 & 0,288 & 0,252 & 0,263 & 0,304 \\
\hline & $\mathrm{p}^{*}$ & 0,9671 & & & 0,5155 & & \\
\hline \multirow{3}{*}{$\begin{array}{l}\text { Atividades do Programa } \\
\text { Saúde na Escola }\end{array}$} & Média & 0,3283 & 0,2512 & 0,453 & 0,2893 & 0,3563 & 0,4411 \\
\hline & DP & 0,322 & 0,229 & 0,283 & 0,288 & 0,277 & 0,323 \\
\hline & $\mathrm{p}^{*}$ & 0,0112 & & & 0,121 & & \\
\hline
\end{tabular}

*ANOVA (Comparação entre os valores das atividades desenvolvidas).

Tabela 4. Taxa médias de homicídio nos últimos 5 e 10 anos, por atividades desenvolvidas ou não pelos Agentes Comunitários de Saúde (ACS) em seu trabalho junto a Estratégia Saúde da Família (ESF). Fortaleza, Ceará, Brasil.

\begin{tabular}{llrr}
\hline \multicolumn{2}{c}{ Atividades dos Agentes Comunitários de Saúde } & $\begin{array}{c}\text { Taxa Homicídio últimos } \\
\mathbf{5} \text { anos }\end{array}$ & $\begin{array}{c}\text { Taxa Homicídio últimos } \\
\mathbf{1 0} \text { anos }\end{array}$ \\
\hline Realiza Visita Domiciliares & Não & 75,61 (DP 48,32) & 82,44 (DP 50,78) \\
& Sim & 63,66 (DP 36,64) & 69,53 (DP 38,55) \\
& $\mathrm{p}^{*}$ & 0,0423 & 0,0371 \\
Atividades de Promoção da Saúde & Não & 61,26 (DP 41,42) & 67,99 (DP 42,24) \\
& Sim & 65,33 (DP 35,61) & $71,33(38,24)$ \\
& $\mathrm{p}^{*}$ & 0,1368 & 0,1314 \\
Atividades do Programa Saúde na & Não & 61,11 (DP 34,22) & 66,90 (DP 36,49) \\
Escola & Sim & 69,06 (DP 41,28) & 75,19 (DP 42,78) \\
& $\mathrm{p}^{*}$ & 0,0014 & 0,0016 \\
\hline
\end{tabular}

${ }^{\star}$ Teste $\mathrm{t}$ (Comparação nas taxas médias - comparando sim ou não).

Do total de 1.239 ACS, a maioria disse que realiza visita domiciliar $(1.181 ; 95,4 \%)$ e atividades de promoção da saúde com grupos específicos (879; 71,1\%). Entretanto, apenas 463 (37,4\%) informam realizar atividades do PSE (Tabela 4). Quando perguntado ao ACS se este se sentia capacitado para lidar com a violência na comunidade em que trabalha, 825 (68,2\%) disseram que não, dos 1.210 que responderam esta pergunta. Para tentar compreender se o gênero, a idade e o tempo como ACS relacionava-se com se sentir preparado para lidar com a violência na comunidade em que trabalha, os testes qui-quadrado e teste $\mathrm{t}$ foram realizados, respectivamente (Tabela 5). Observou-se que ACS do gênero masculino e aqueles com maior idade se sentem mais preparados para lidar com a violência.

\section{Discussão}

Os resultados deste estudo evidenciam um padrão bastante heterogêneo em relação à disseminação de casos e óbitos por COVID-19 em Fortaleza, considerando-se os distintos territórios de atuação dos ACS e seus perfis de violência e vulnerabilidade social, o que reflete a complexidade e os desafios no enfrentamento da pandemia em grandes centros urbanos, marcados por grandes desigualdades sociais. Observa-se uma relação 
Tabela 5. Avaliação da relação entre o gênero, a idade, e o tempo como ACS com o se sentir preparado para lidar com a violência na comunidade em que trabalha. Fortaleza, Ceará, Brasil.

\begin{tabular}{|c|c|c|}
\hline Variáveis avaliadas & Resultado do teste estatístico & $\begin{array}{c}\text { Interpretação do resultado } \\
\text { estatístico }\end{array}$ \\
\hline $\begin{array}{l}\text { ACS se sente capacitado para lidar } \\
\text { com a violência na comunidade } \\
\text { em que trabalha } v s \text { Idade }\end{array}$ & $\begin{array}{l}\text { Há relação - p <0,001 (Two sample } \\
\text { ttest) }{ }^{*}\end{array}$ & $\begin{array}{l}\text { ACS mais velhos se sentem mais } \\
\text { capacitados a lidar com a violência na } \\
\text { comunidade em que trabalham }\end{array}$ \\
\hline $\begin{array}{l}\text { ACS se sente capacitado para lidar } \\
\text { com a violência na comunidade } \\
\text { em que trabalha } v s \text { Tempo como } \\
\text { ACS }\end{array}$ & $\begin{array}{l}\text { Há tendência a relação - p=0,0921 } \\
\text { (Two sample ttest)* }\end{array}$ & $\begin{array}{l}\text { Tendência a ACS com mais tempo } \\
\text { de profissão se sentirem mais } \\
\text { capacitados a lidar com a violência na } \\
\text { comunidade em que trabalha }\end{array}$ \\
\hline $\begin{array}{l}\text { ACS se sente capacitado para lidar } \\
\text { com a violência na comunidade } \\
\text { em que trabalha vs Gênero }\end{array}$ & $\begin{array}{l}\text { Há relação - p=0,003 (teste Chi- } \\
\text { Square)** }\end{array}$ & $\begin{array}{l}\text { Homens se sentem mais preparados } \\
\text { que as mulheres para lidar com a } \\
\text { violência }\end{array}$ \\
\hline
\end{tabular}

${ }^{*}$ Teste $\mathrm{t} ;{ }^{* *}$ Teste qui-quadrado.

negativa dos casos de COVID-19 com indicadores de vulnerabilidade social (e.g., IDH e analfabetismo), e uma relação positiva dos óbitos de COVID-19 com indicadores de vulnerabilidade social (e.g., homicídios, cobertura do Programa Bolsa Família). No entanto, a atuação do ACS, no enfrentamento do COVID-19, é limitada pela violência do território.

Aparentemente, os casos são mais frequentes em regiões menos vulneráveis, enquanto a mortalidade é maior nas regiões de maior vulnerabilidade. Mas, apesar de fazer sentido o número de casos ser maior nas regiões menos vulneráveis no início da pandemia em Fortaleza, haja vista que as evidências apontam que ela "aterrissou" na capital cearense trazida por viajantes internacionais, de poder aquisitivo mais alto e residentes em bairros de classe média-alta, imaginava-se que, com a migração da doença para as regiões mais pobres do município, ocorrida desde abril de 2020 (boletim epidemiológico de Fortaleza, https://coronavirus.fortaleza.ce.gov.br/boletim -epidemiologico.html), a relação de casos de COVID-19 com indicadores de vulnerabilidade fosse se invertendo. Dados de outras pesquisas indicam o alto risco para casos de COVID-19 em regiões de alta vulnerabilidade social ${ }^{6}$.

Entretanto, isto não é o que os dados aqui apresentados demonstram. A explicação para esta aparente incongruência não é clara, mas pode estar relacionada a uma taxa de testagem mais baixa nas regiões de maior vulnerabilidade. Ademais, se a hipótese de baixa testagem nessas regiões for verdadeira, ela compromete, também, o enfrentamento da pandemia nessas comunidades, deixando-as ainda mais susceptíveis à disseminação da pandemia. Infelizmente, não temos informações sobre taxa de testagem por bairro e/ ou região de saúde de Fortaleza para comprovar essa hipótese.

A relação de taxa de óbitos de COVID-19 com vulnerabilidade social não é novidade na atual pandemia. Dados dos municípios de Manaus e Rio de Janeiro também demonstraram correlações semelhantes ${ }^{3,6}$. Casos graves de COVID-19 no Rio de Janeiro ocorrem em áreas de vulnerabilidade de toda a cidade, mas, principalmente, nas periferias e nas comunidades carentes incrustadas em áreas nobres ${ }^{6}$. Em Manaus, o impacto da mortalidade é mais acentuado nas áreas com condições socioeconômicas mais precárias ${ }^{3}$.

De modo geral, os dados aqui apresentados demonstram que os óbitos por COVID-19 se distribuíram por todo o território do município de Fortaleza, mas observou-se maior crescimento e concentração em bairros onde variáveis sociais, como cobertura do PBF e taxa de homicídios são elevados. Denota-se que a disseminação da morbimortalidade por doenças infecciosas é um processo espacial que pode ter distribuição heterogênea, mas se deve considerar a possibilidade de maior potencial de contágio em áreas vizinhas, decorrente da proximidade e do aumento dos laços socioculturais ${ }^{19}$. Compreender esse padrão espacial é essencial para identificar quem está em maior risco e para alocar recursos e respostas adequadas. As desigualdades sociais aumentam quando confrontadas com doenças epidêmicas, os fatores econômicos constituem desvantagem social, que aumenta a vulnerabilidade diante da rápida disseminação das doenças infecciosas ${ }^{20}$.

Apesar de Fortaleza acumular, historicamente, processos e movimentos de lutas em favor da construção de políticas públicas, particularmen- 
te de educação, de saúde e de geração de renda, desenvolvidas por iniciativa dos movimentos sociais, com vistas a minimizar a disparidade socioeconômica existente, sobretudo na grande periferia da cidade ${ }^{21,22}$, o município foi considerado, em 2012, a segunda cidade brasileira de maior desigualdade social ${ }^{21}$. Ressalta-se o fato de que a capital cearense tem um cenário socioespacial heterogêneo, inclusive com bairros de classe média-alta limítrofes com bairros de extrema pobreza. Nesses, predominam o saneamento básico precário, a alta densidade populacional e a dificuldade de acesso a bens e serviços. Ademais, a cultura socioeconômica põe em disputa o microempreendedor e os grandes empresários do setor de negócios e de turismo. Vale ressaltar que mais da metade da população brasileira vive com menos de um salário mínimo ou na informalidade. Nesse contexto, com as medidas de quarentena em todos os estados da federação, agrava-se o perfil de vulnerabilidade social dessas pessoas, pois podem não ter renda, nem acesso à água em suas residências e/ou simplesmente não compreenderem quais são as medidas de prevenção indicadas contra a COVID-19².

A maior cobertura do PBF nas áreas mais vulneráveis da capital cearense pode indicar o alcance desta política junto à grande parcela da população com baixas condições socioeconômicas. Assim, esta política de redistribuição de renda impacta no bem-estar das famílias em condições de pobreza. Desde sua implantação, o PBF tem como diretrizes o combate à pobreza e à fome no país, tal como a promoção de inovações na intervenção pública na área social ${ }^{24}$. Com efeito, tem cumprido relevante papel na promoção da justiça social e equidade no Estado brasileiro, melhorando relativamente as condições de vida e saúde da população ${ }^{25}$. Porém, parece que, isoladamente, não consegue equacionar as históricas desigualdades sociais, o que requer sua integração com uma ampla e efetiva política de proteção social que assegure o acesso universal ao trabalho, à renda, à educação, à segurança pública e à saúde.

O cenário desafiador da pandemia no Brasil tem posto em evidência não somente a problemática das desigualdades sociais, mas também a capacidade de resposta do sistema de saúde, sob constante ameaça de colapso ante o aumento de casos da doença, seja pela insuficiência de respiradores e de leitos de $\mathrm{UTI}^{26}$; seja pelas limitações nas ações de prevenção e controle, particularmente o isolamento social ${ }^{27}$; seja, sobretudo, pela atuação de lideranças políticas que subestimam os impactos e adotam posturas negacionistas ${ }^{28}$.
Soma-se a isso, o fato de que o SUS enfrenta problemas conjunturais históricos, que se configuram como condicionantes prévios ao cenário atual, relacionados ao subfinanciamento, à baixa institucionalidade da capacidade de gestão, à falta de investimento na ampliação da atenção especializada e à redução de leitos na estrutura hospitalar, além dos processos de privatização em curso promovidos pelas contrarreformas do Estado brasileiro, que têm efeitos nefastos na garantia da efetividade do direito à saúde da população ${ }^{29}$.

Todavia, observa-se que no SUS, em meio aos impactos das políticas neoliberais de implantação do Estado mínimo, que culminam em processos de privatização, além da ausência da atuação coordenada do Governo Federal, os gestores e os trabalhadores de saúde, nos âmbitos estadual e local, têm desenvolvido Planos de Contingência da COVID-19, gerenciado os fluxos assistenciais e buscado suprir a necessidade de insumos, de medicamentos, de material e de equipamentos, particularmente de proteção individual ${ }^{30}$. Destaca-se, ainda, a estrutura altamente qualificada da vigilância à saúde responsável pelo monitoramento dos casos e proposição de medidas de controle e de mitigação dos efeitos. Além disso, as propostas de expansão de cobertura das ações da APS e o estabelecimento de diretrizes clínicas para atuação das equipes nos distintos níveis de atenção, onde se definiu as competências dos profissionais, incluindo-se aí o ACS, que tem papel fundamental no enfrentamento da COVID-19 $9^{12,13}$.

Apesar da capacidade instalada e dos esforços governamentais, a emergência sanitária imposta pela pandemia sobrecarrega os serviços de saúde, muitas vezes com equipes reduzidas e condições mínimas de trabalho, o que acaba por reduzir o acesso e a oferta de tratamento para os demais agravos, corroborando para o aprofundamento das vulnerabilidades. Com efeito, a pandemia da COVID-19 agrava as condições de saúde das populações vulneráveis, sobrepondo-se a carga de doenças que apresentam maior prevalência nesse segmento populacional, principalmente as doenças negligenciadas, como tuberculose, hanseníase, sífilis, entre outras ${ }^{4,31}$. Desse modo, as equipes de saúde do território são desafiadas a equilibrar a oferta de cuidados às pessoas no âmbito das ações programáticas e aos indivíduos com agravos agudos e emergentes. Destarte, nos casos de COVID-19, a APS deve atuar fortemente nas orientações para a prevenção e no monitoramento dos casos leves, conforme as diretrizes clínicas e as necessidades de saúde identificadas no território. 
Regiões de maiores taxas de óbito por COVID-19 e maior vulnerabilidade social são as mesmas com maior cobertura da ESF. Contudo, estas também são as regiões onde o ACS menos realiza visita domiciliar e, proporcionalmente, mais realiza atividades no PSE. O aumento das atividades junto ao PSE deve-se, possivelmente, ao tempo não utilizado para visita domiciliar, assim como pela atuação do ACS em local de violência mais controlada (escola), quando comparado à comunidade como um todo. $\mathrm{O}$ motivo para a menor atuação em visitas domiciliares no território ocorre, provavelmente, devido às taxas de violência da região, que, por sua vez, estão comumente relacionadas aos indicadores de vulnerabilidade, e retroalimentam a violência no território, limitando a atuação do ACS. Tradicionalmente, estes são os territórios onde as ações do Estado têm dificuldade em chegar, territórios de governança limitada, e onde outros desafios sanitários precisam ser enfrentados.

Todavia, é importante lembrar que os dados primários do presente trabalho foram coletados antes do início da pandemia, demonstrando que a influência da violência no processo de trabalho do ACS é anterior à onda de violência doméstica e comunitária vista com a chegada do COVID19 $9^{7-9,32}$. Assim, é possível que o impacto da violência no processo de trabalho do ACS seja ainda maior do que o apresentado na presente pesquisa. Desse modo, esforços devem ser direcionados à ampliação das ações das equipes da ESF na linha de frente no âmbito da prevenção, à ampla divulgação dos serviços disponíveis, à capacitação dos trabalhadores da saúde para identificar situações de risco de violência, bem como a expansão e o fortalecimento das redes de apoio às vítimas ${ }^{32}$.

Compreendendo o papel dos ACS na promoção da saúde e na prevenção de agravos, reitera-se a importância de sua atuação no enfrentamento da COVID-19. Este, habitualmente, mora na mesma comunidade em que trabalha, tem conhecimento profundo do território coberto pela ESF, o que facilita a identificação e o monitoramento de casos. Parece-nos, entretanto, que a realidade concreta no território, incluindo a violência, impõe a não execução efetiva das diretrizes de trabalho dos ACS, essencialmente voltadas à educação em saúde, promoção da saúde e à realização de visitas domiciliares, ações fundamentais na atuação da APS brasileira no enfrentamento à COVID-1933.

A plena atuação do ACS, que tem grande potencial no enfrentamento da COVID-19, é dependente de políticas intersetoriais. Assim, a construção de uma política de enfrentamento da COVID-19, com efetiva participação do ACS, precisa levar em consideração, além do treinamento adequado deste profissional em medidas de prevenção e detecção de casos da doença, ações intersetoriais voltadas à prevenção e controle da violência no território.

O enfrentamento multifacetado das vulnerabilidades sociais parece ser a forma mais efetiva de atuação do Estado, não somente a atual pandemia, mas também noutras questões de saúde que afetam estas populações. O Estado precisa prover saúde, segurança, oportunidades educacionais, condições de acesso ao trabalho, acesso ao lazer e cultura, tal como desenvolvimento comunitário, materializando, assim, uma ampla política de proteção/seguridade social, que deve incluir, mas não se limitar a programas de transferência de renda (e.g., Programa Bolsa Família). Nesse contexto, é imprescindível assegurar o fortalecimento da APS e de sistemas universais de saúde, o que reforça a necessidade de luta em defesa SUS. 


\section{Colaboradores}

Todos os autores participaram da concepção e revisões que resultaram neste artigo.

\section{Referências}

1. World Health Organization (WHO). Coronavirus disease 2019 (COVID-19). Situation report-87. Geneva: WHO; 2020.

2. Brasil. Ministério da Saúde (MS). Covid-19: painel coronavirus [Internet]. [acessado 2020 Jul 18]. Disponível em: https://covid.saude.gov.br/

3. Orellana JDY, Cunha GM, Marrero L, Horta BL, Leite IC. Explosão da mortalidade no epicentro amazônico da epidemia de COVID-19. Cad Saúde Pública 2020; 36(7):e00120020.

4. Goes EF, Ramos DO, Ferreira AJF. Desigualdades raciais em saúde e a pandemia da Covid-19. Trab Educ Saúde 2020; 18(3):e00278110.

5. Souza ER, Njaine K, Ribeiro AP, Legay L, Meira KC. Violência estrutural e COVID-19 [Internet]. Abrasco; 2020 [acessado 2020 Jul 14]. Disponível em: https://www.abrasco.org.br/site/gtviolenciaesaude/ 2020/05/19/violencia-estrutural-e-covid-19/

6. Santos JPC, Siqueira ASP, Praça HLF, Albuquerque HG. Vulnerabilidade a formas graves de COVID-19: uma análise intramunicipal na cidade do Rio de Janeiro, Brasil. Cad Saúde Pública 2020; 6(5):e00075720.

7. Marques ES, Moraes CL, Hasselmann MH, Deslandes SF, Reichenheim ME. A violência contra mulheres, crianças e adolescentes em tempos de pandemia pela COVID-19: panorama, motivações e formas de enfrentamento. Cad Saúde Pública 2020; 36(4):e00074420.

8. Núcleo de Estudos da Violência. Universidade de São Paulo. Qual o impacto da pandemia nos índices de criminalidade [Internet]. [acessado $2020 \mathrm{Jul} \mathrm{16].}$ Disponível em: https://nev.prp.usp.br/imprensa/ qual-o-impacto-da-pandemia-nos-indices-de-criminalidade/

9. Bertoni E. Qual o impacto da pandemia nos indices de criminalidade? [Internet]. [acessado $2020 \mathrm{Jul}$ 15]. Disponível em: https://www.nexojornal.com. br/expresso/2020/04/14/Qual-o-impacto-da-pandemia-nos-\%C3\%ADndices-de-criminalidade

10. Guimarães C. A importância de um sistema de saúde público e universal no enfrentamento à epidemia [Internet]. [acessado 2020 Jul 17]. Disponível em: http:// www.epsjv.fiocruz.br/noticias/reportagem/a-importancia-de-um-sistema-de-saude-publico-e-universal-no-enfrentamento-a.

11. Brasil. Ministério da Saúde (MS). Portaria no 2.436, de 21 de setembro de 2017. Aprova a Política Nacional de Atenção Básica, estabelecendo a revisão de diretrizes para a organização da Atenção Básica, no âmbito do Sistema Único de Saúde (SUS). Diário Oficial da União 2017; 22 set.

12. Brasil. Ministério da Saúde (MS). Protocolo de manejo clínico do coronavírus (Covid-19) na atenção primária à saude. Versão 9 [Internet]. Brasília: MS; 2020 [acessado 2020 Jul 14]. Disponível em: https://www.unasus.gov.br/especial/covid19/pdf/37

13. Brasil. Ministério da Saúde (MS). Orientações gerais sobre a atuação do ACS frente à pandemia de COVID-19 e os registros a serem realizados no e-SUS APS [Internet]. Brasília: MS; 2020 [acessado 2020 Jul 18]. Disponívem em: http://189.28.128.100/dab/ docs/portaldab/documentos/esus/Orientacoes_ACS_ COVID_19.pdf 
14. Nascimento TMRV, Resnicow K, Nery M, Brentani A, Kaselitz E, Agrawal P, Mand S, Heisler M. A pilot study of a Community Health Agent-led type 2 diabetes self-management program using Motivational Interviewing-based approaches in a public primary care center in São Paulo, Brazil. BMC Health Serv Res 2017; (17):32.

15. Fortaleza. Secretaria Municipal de Saúde de Fortaleza (SMS-FORTALEZA). COVID-19: informe semanal $28^{a}$ semana epidemiológica [Internet]. [acessado 2020 Jul 10]. Disponível em: https://coronavirus.fortaleza. ce.gov.br/boletim-epidemiologico.html

16. Brasil. Ministério da Saúde (MS). Atenção primária à saúde: painel de indicadores [Internet]. [acessado 2020 Jul 13]. Disponível em: https://sisaps.saude.gov.br/ painelsaps/saude-familia

17. Brasil. Instituto de Pesquisa Econômica Aplicada (IPEA). Atlas da violência: Retratos dos municípios brasileiros - 2019 [Internet]. Rio de Janeiro: IPEA; 2019 [acessado 2020 Jul 12]. Disponível em: https://www. ipea.gov.br/portal/index.php?option=com_content\&view $=$ article\&id $=34943 \&$ Itemid $=432$

18. Seguridad, Justicia y Paz. Las 50 ciudades más violentas del mundo 2018 [Internet]. México; 2019 [acessado 2020 Jul 10]. Disponível em: http://seguridadjusticiaypaz.org.mx/files/estudio.pdf .

19. Corde J, Castro MC. Spatial analysis of COVID-19 clusters and contextual factors in New York City. Spatial Spatio-Temporal Epidemiol 2020; 34:100355.

20. Van Barvel JJ, Baicker K, Boggio PS, Capraro V, Cichocka A, Cikara M, Crockett MJ, Crum AJ, Douglas KM, DruckmanJN, Drury J, Dube O, Ellemers N, Finkel EJ, Fowler JH, Gelfand M, Han S, Haslam AS, Jetten J, Kitayama S, Mobbs D, Napper LE, Packer DJ, Pennycook G, Peters E, Petty RE, Rand DG, Reicher SD, Schnall S, Shariff A, Skitka LJ, Smith SS, Sunstein CR, Tabri N, Tucker JA, Linden S, Lange P, Weeden KA, Wohl MJA, Zaki J, Zion SR, Willer R. Using social and behavioural science to support COVID-19 pandemic response. Nat Hum Behav 2020; 4:460-471.

21. Araújo RV, Costa MCL. Da migração sertaneja ao surgimento das favelas: a formação socioespacial e vulnerabilidade em Fortaleza, Ceará. Geosaberes 2015; 6(3):585-598.

22. Ribeiro KG, Andrade LOM, Aguiar JB, Moreira AEMM, Frota AC. Educação e saúde em uma região em situação de vulnerabilidade social: avanços e desafios para as políticas públicas. Interface (Botucatu) 2018; 22(Supl. 1):1387-1398.

23. Silva DN. Determinantes sociais da vulnerabilidade à Covid-19: proposta de um esquema teórico - Parte II [Internet]. Unifesspa contra a Covid-19. [acessado 2020 Jul 20]. Disponível em: https://acoescovid19. unifesspa.edu.br/images/Artigo_-_Parte_2_-_Completo.pdf

24. Burlandy L. Transferência condicionada de renda e segurança alimentar e nutricional. Cien Saude Colet 2007; 12 (6):1441-1451.
25. Vieira-Meyer APGF, Dias MSA, Vasconcelos MIO, Rouberte ESC, Almeida AMB, Pinheiro TXA, Saintrain MVL, Machado MFAS, Dufault S, Reynolds SA, Fernald L. What is the relative impact of primary health care quality and conditional cash transfer program in child mortality? Can J Public Health 2019; 110(6):756-767.

26. Noronha KVMS, Guedes GR, Turra CM, Andrade MV, Botega L, Nogueira D, Calazans JA, Carvalho L, Servo L, Ferreira MF. Pandemia por COVID-19 no Brasil: análise da demanda e da oferta de leitos hospitalares e equipamentos de ventilação assistida segundo diferentes cenários. Cad Saúde Pública 2020; 36(6):e00115320.

27. Andrade BBP, Peres IT, Baião FA, Ranzani OT, Bastos LSL, Silva AAB, Souza GFG, Marchesi JF, Dantas LF, Vargas SA, Maçaira P, Hamacher S, Bozza FA. Progressão dos casos confirmados de COVID-19 após implantação de medidas de controle. Rev Bras Ter Intensiva 2020; 32(2):213-223.

28. Campos GWS. O pesadelo macabro da Covid-19 no Brasil: entre negacionismos e desvarios. Trab Educ Saúde 2020; 18(3):e00279111.

29. Dantas AV. Coronavírus, o pedagogo da catástrofe: lições sobre o SUS e a relação entre público e privado. Trab Educ Saúde 2020; 18(3):e00281113.

30. Araújo JL, Oliveira KKD, Freitas RJM. Em defesa do Sistema Único de Saúde no contexto da pandemia por SARS-CoV-2. Rev Bras Enferm 2020; 73(Supl. 2):e20200247.

31. Carvalheiro JR. Os coletivos da Covid-19. Estud Av 2020; 34(99):7-24

32. Vieira PR, Garcia LP, Maciel EL. Isolamento social e o aumento da violência doméstica: o que isso nos revela? Rev Bras Epidemiol 2020; 23:e200033.

33. Harzheim E, Santos CMJ, D’Avila Pereira O, Wollmann L, Pinto LF. Bases para a Reforma da Atenção Primária à Saúde no Brasil em 2019: mudanças estruturantes após 25 anos do Programa de Saúde da Família. Rev Bras Med Fam Comunidade 2020; 15(42):2354.

Artigo apresentado em 08/08/2020

Aprovado em 30/10/2020

Versão final apresentada em $01 / 11 / 2020$

Editores chefes: Romeu Gomes, Antônio Augusto Moura da Silva 